\title{
Effect of annealing and cobalt content on relaxation and crystallization behavior of zirconium based bulk metallic glasses
}

\author{
Yue Dong ${ }^{1 *}$, Rainer Wunderlich ${ }^{1}$, and Hans-Jörg Fecht ${ }^{1}$ \\ ${ }^{1}$ Institute of Micro and Nanomaterials, University of Ulm, Albert-Einstein Allee 47, $89081 \mathrm{Ulm}$, \\ Germany
}

\begin{abstract}
The effects of annealing and cobalt content on relaxation and the crystallization process of $\mathrm{Zr}_{64} \mathrm{Ni}_{10} \mathrm{Al}_{7} \mathrm{Cu}_{19}$ bulk metallic glasses were investigated. $\beta$-relaxation occurs during annealing, leading to increased endotherm before crystallization. $\alpha$-relaxation during high temperature annealing (higher than $\mathrm{Tg}$ ) affects the crystallization process. The introduction of cobalt leads to an inhomogeneous amorphous structure and two-step crystallization due to the positive mixing enthalpy between cobalt and copper. Non-affine thermal strain arising from low temperature annealing of heterogeneous structure leads to a reduced endotherm phenomenon during relaxation on the DSC curves and a reduction in hardness.
\end{abstract}

\section{Introduction}

Metallic glasses are amorphous alloys, meaning they are disordered at the atomic scale. Different from standard metals, metallic glasses have no long-range order. Due to their unique structures, including a lack of grain boundaries or dislocations, bulk metallic glasses often exhibit excellent properties, including high strength, high hardness, low Young's modules and good corrosion resistance. However, metallic glasses are still not widely used. Although metallic glasses have been investigated for many years, the amorphous structure is still not well understood [1].

To produce a metallic glass, the metallic liquid needs to be cooled down without any nucleation. Thus, the properties of the supercooled liquid are correlated with the structure and properties of the formed metallic glass [2,3]. Due to their metastability, the relaxation and crystallization behaviors of the supercooled liquids and glassy solids are related to their structures and properties. It has been shown that activation energy of the relaxation is nearly equivalent to the activation energy of shear transformation zones, and the temperature difference between crystallization and relaxation has been shown to be related to the metal's glass forming ability [4]. Furthermore, the study of metallic glasses is vital to understanding the nature of glass. However, the factors that govern relaxation and

\footnotetext{
* Corresponding author: yue.dong@,uni-ulm.de
} 
crystallization are still unclear and are thus actively and systematically being investigated $[5,6,7]$.

Zr-based bulk metallic glasses have been the subject of intensive research because of their good glass forming ability and excellent mechanical properties [8]. W Wang et al. [9] investigated the $\mathrm{Zr}-\mathrm{Cu}-\mathrm{Ni}-\mathrm{Al}$ system and found that for some compositions, the metallic glasses show good plasticity. In this work, we will focus on effects of annealing and composition on the relaxation and crystallization processes of this alloy series.

\section{Experimental methods}

Ingots of different compositions $\left(\mathrm{Zr}_{64-\mathrm{x}} \mathrm{Ni}_{10} \mathrm{Al}_{7} \mathrm{Cu}_{19} \mathrm{Cox}, \mathrm{x}=0-8.3\right.$ at.\%) were prepared by arcmelting a mixture of high purity $\mathrm{Zr}, \mathrm{Ni}, \mathrm{Al}, \mathrm{Cu}$ and $\mathrm{Co}$ elements in a Ti-gettered argon atmosphere. The as-obtained ingots were then melted and sucked into a water-cooled copper mold. The samples were glassy rods $4 \mathrm{~mm}$ in diameter and $40 \mathrm{~mm}$ in length. The structure of the as-casted samples were analyzed by X-ray diffraction (XRD) with $\mathrm{Cu}-\mathrm{K} \alpha$ radiation. Thermal analysis was carried out in a differential scanning calorimeter under a high purity Ar flow. In continuous heating, the scan rate was $20 \mathrm{~K} / \mathrm{min}$ from $373 \mathrm{~K}$ to $830 \mathrm{~K}$. Each sample was heated twice so that the baseline could be obtained from the second run and subtracted to get the final curve. For the isothermal annealing, the samples were first heated to a fixed temperature with a fast heating rate $(160 \mathrm{~K} / \mathrm{min})$ from room temperature and then held. The results can also be used to calculate the heat flow as a function of time. To monitor changes in the structure of the BMGs, selected samples were analyzed with a transmission electron microscope (TEM), a Philips CM20 operating at 200kV. Electron transparent TEM lamellae were produced by mechanical grinding and polishing followed by low angle Ar-ion milling. To investigate the change in hardness, nanoindentation measurements were completed with a Berkovich indenter on the polished samples.

\section{Results}

Fig. 1 shows the XRD results for samples with different cobalt contents. All of the samples have fully amorphous structures. With increasing cobalt content, the diffraction maxima shift to the right, indicating that the sample with the highest cobalt content has the smallest interatomic distance due to cobalt having smaller atom diameter in comparison to zirconium.

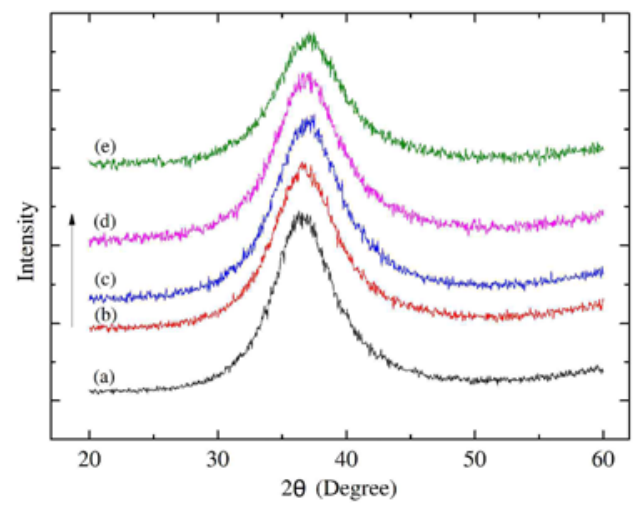

Fig. 1. X-ray diffraction patterns for $\mathrm{Zr}_{64-\mathrm{x}} \mathrm{Ni}_{10} \mathrm{Al}_{7} \mathrm{Cu}_{19} \mathrm{CO}_{x}$ as a function of the Co content after casting: (a) $0 \%$, (b) $2.7 \%$, (c) $4 \%$, (d) $6 \%$, (e) $8.3 \%$ 
To study the effect of annealing on the relaxation process, the $\mathrm{Zr}_{64} \mathrm{Ni}_{10} \mathrm{Al}_{7} \mathrm{Cu}_{19}$ alloys were annealed at different temperature $606 \mathrm{~K}-658 \mathrm{~K}$ (Tg for this alloy is $648 \mathrm{~K}$.). Fig. 2(a) shows that annealing introduces an endothermic peak at the beginning of the relaxation on the DSC curves. The peak intensity increases at first and then reduces with the rising annealing temperature. This peak also shifts to a higher temperature with the rising annealing temperature. Furthermore, in Fig. 2(b), it shows that with the rising annealing temperature, the peak intensity is reduced, the peak width increases and the crystallization peak shifts to a lower temperature when the annealing temperature is higher than $\mathrm{Tg}$.
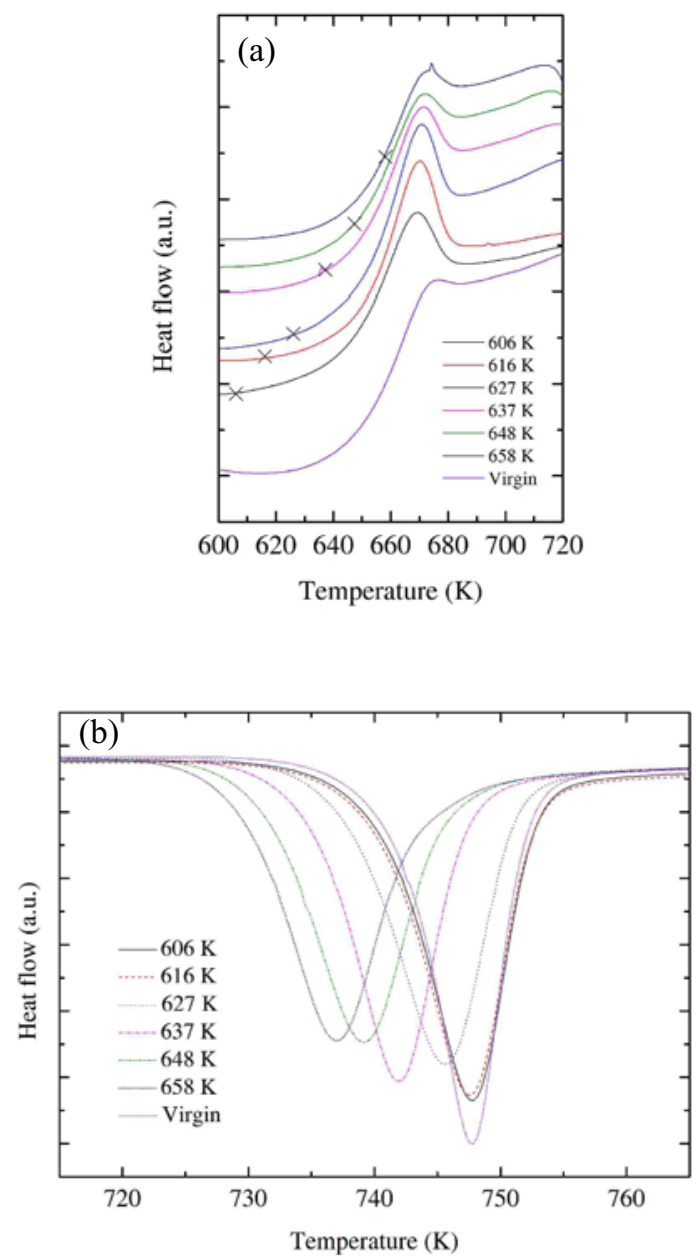

Fig. 2. (a) Changes in the heat flow around $\mathrm{Tg}$ on the DSC curves of $\mathrm{Zr}_{64} \mathrm{Ni}_{10} \mathrm{Al}_{7} \mathrm{Cu}_{19} \mathrm{BMGs}$ annealed at different temperatures. The cross on the curve is the annealing temperature before the DSC measurement. (b) Changes in the crystallization peak on the DSC curves for $\mathrm{Zr}_{64} \mathrm{Ni}_{10} \mathrm{Al}_{7} \mathrm{Cu}_{19}$ BMGs annealed at different temperature.

Fig. 3(a) shows the continuous heating DSC curves for alloys with different cobalt contents. The DSC curves show that the cobalt composition affects the thermal properties of the alloys so dramatically that the crystallization peak splits into two peaks. With the increasing cobalt content, the distance between the two peaks becomes larger, the intensity 
of the first peak increases, and the second peak exhibits a corresponding decrease so that the total enthalpy remains consistent. In Fig. 3 (b), it can be seen that the glass transition temperature $\mathrm{Tg}$ shifts to a higher temperature due to the increase in cobalt content. The temperature range of the relaxation (between $\mathrm{Tg}$ and $\mathrm{Tx}$ ) also reduces with increasing cobalt content.
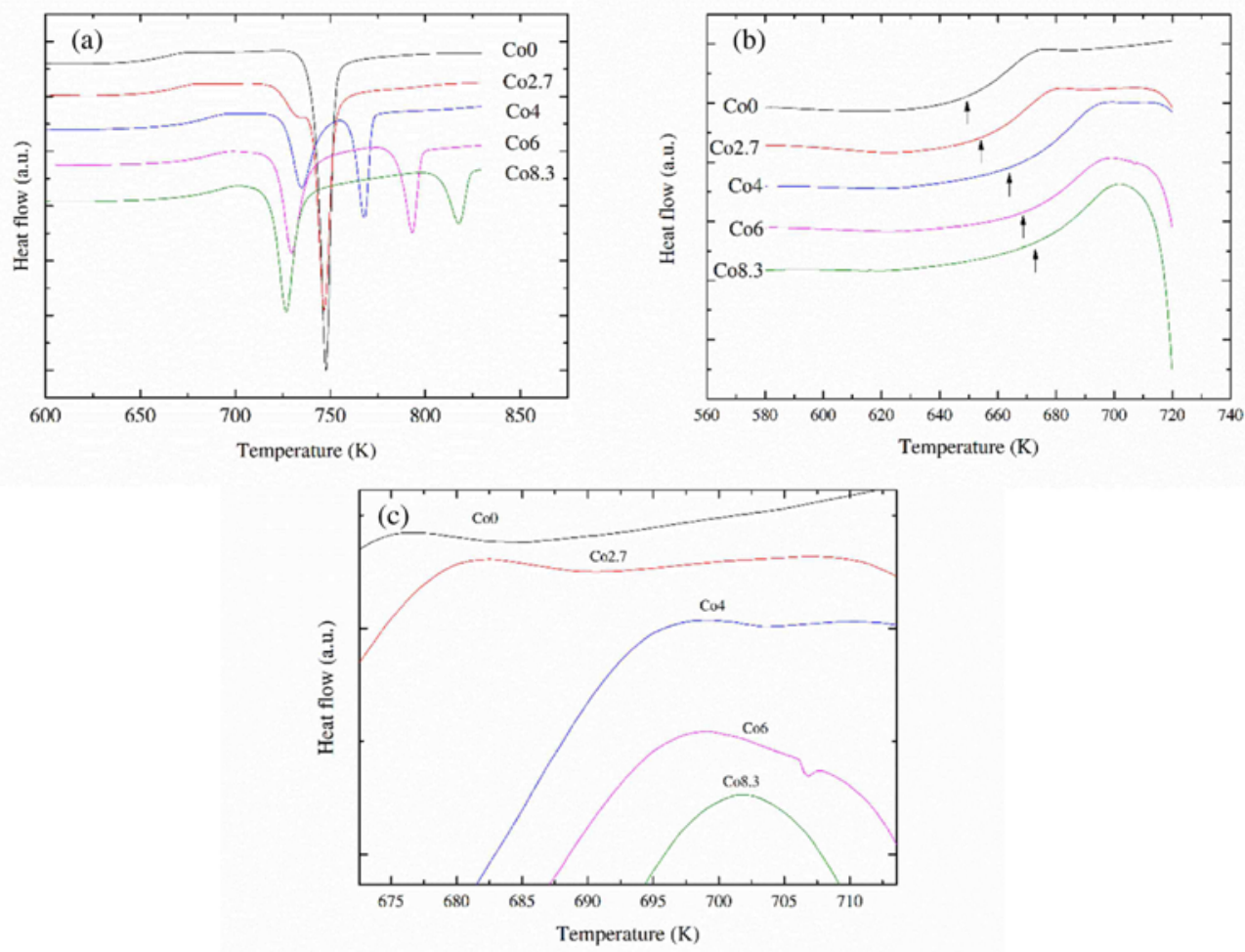

Fig. 3. (a) DSC curves of the BMGs with different cobalt content. (b) Change of $\mathrm{Tg}$ as a function of Co content $(0 \%, 2.7 \%, 4 \%, 6 \%, 8.3 \%)$. (c) Change in the relaxation properties as a function of Co content as measured by DSC

Fig. 4 shows the DSC curves of samples with various Co contents annealed at different temperatures. When the samples that contained Co were annealed at a lower temperature $(563 \mathrm{~K})$, a special endotherm reducing event occurs before relaxation, as shown in the DSC measurement Fig. 4 (d). Furthermore, with increasing of Co content, the presence of the endotherm reducing event becomes more obvious. Whereas, when the samples were annealed at a higher temperature $(583 \mathrm{~K})$, there was no substantial change from the results of samples without Co. However, the height of the endothermic peak before relaxation is reduced with the addition of Co. 

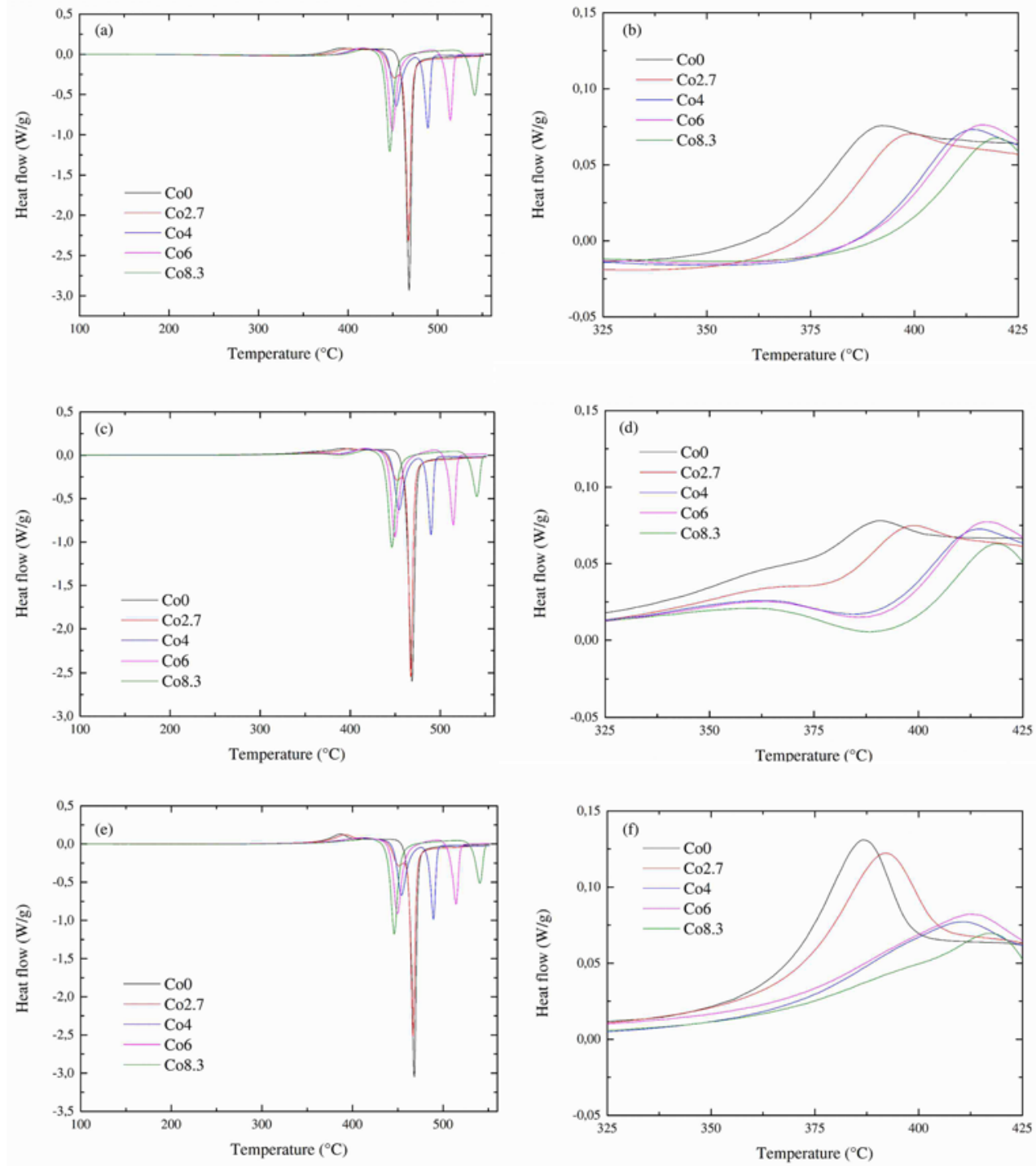

Fig. 4. (a) DSC curves of the as-cast BMGs with different cobalt contents. (b) The relaxation peak as a function of cobalt content for the as-cast BMGs. (c) DSC curves of the BMGs with different cobalt contents annealed at $563 \mathrm{~K}$. (d) Relaxation of the BMGs with different cobalt contents annealed at $563 \mathrm{~K}$. (e) DSC curves of the BMGs with different cobalt contents annealed at $583 \mathrm{~K}$. (f) Relaxation of the BMGs with different cobalt contents annealed at $583 \mathrm{~K}$.

\section{Discussion}

The XRD results show that the introduction of cobalt does not dramatically reduce the glass forming ability. However, if the Co content is too high (more than 10\%), the BMG will be partially crystallized. Due to the brittle nature of the intermetallic compound formed at high 
cobalt contents, we focus only on the composition range that results in a fully amorphous structure for this BMG (i.e., with Co content between 0 and 8.3\%). From the shift in the diffraction maxima it can be inferred that with increasing cobalt content, the average interatomic distance is reduced, resulting in a stronger atomic interaction.

To investigate the effect of annealing on the relaxation process, $\mathrm{Zr}_{64} \mathrm{Ni}_{10} \mathrm{Al}_{7} \mathrm{Cu}_{19}$ BMGs were first annealed at a temperature around Tg. An endothermic peak appears on the DSC curves prior to relaxation. The peak intensity increases with elevation of the annealing temperature while it is below $\mathrm{Tg}$; however, the peak intensity starts to decrease at annealing temperatures higher than Tg, as shown in Fig. 2(a). This phenomenon has been previously observed in the literature [10]. The energy landscape model can be used to explain this phenomenon [11]. The $\beta$-relaxation determines the features of the $\alpha$-relaxation by acting as a precursor to the glass transition. When the temperature is below $\mathrm{Tg}, \beta$-relaxation is dominant (Fig. 5(b)). As shown in Fig. 5(a), with higher annealing temperatures, the high energy barriers present can be surmounted and thus, the system arrives at a configuration with a lower energy (deeper valley on energy landscape). Then, after annealing, the $\alpha$ relaxation in the following DSC measurement needs higher activation energy to surmount the initial energy barriers. In this way, the heat flow increases and the peak shifts to a higher temperature. If the annealing temperature is higher than $\mathrm{Tg}, \alpha$-relaxation will occur, accompanied by $\beta$-relaxation during annealing, such that the endothermic peak intensity on DSC curve is reduced. In another words, the low temperature $\beta$-relaxation results in a structure with lower energy, which indicates denser atomic packing. Thus, the low temperature $\beta$-relaxation leads to a higher diffusion energy for the following $\alpha$-relaxation, resulting in the appearance of the endothermic peak prior to relaxation on the DSC curves; alternatively, when the annealing temperature is higher than $\mathrm{Tg}$, the diffusion energy is reduced.

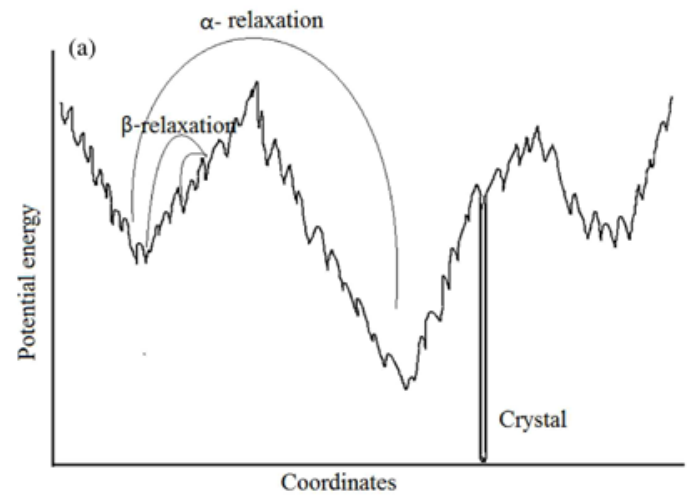

(b)

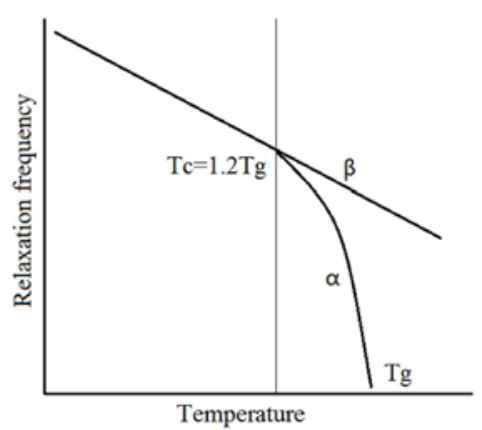

Fig. 5. Schematic illustration of (a) an energy landscape, (b) relaxation frequency change as a function of temperature [11]

The difference between low and high temperature annealing (lower or higher than $\mathrm{Tg}$, respectively) can also be found in Fig. 6. There is an apparent difference in heat flow during annealing. The curves can be well fitted with the equation $W(t)=W_{0}\left(1-e^{t / \tau}\right)$, where $W_{0}$ is the increase in heat flow $W(t)$ after an infinite time and $\tau$ is the relaxation time. The fit parameters are given in Table 1. 


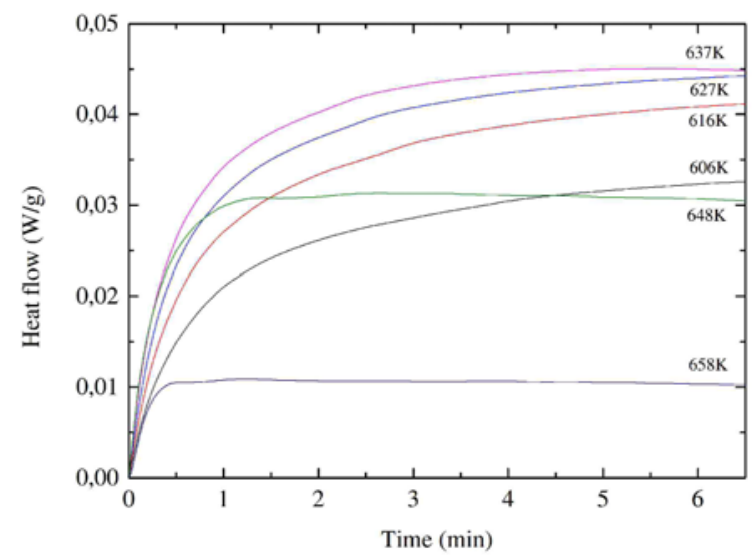

Fig. 6. Heat flow on $\mathrm{Zr}_{64} \mathrm{Ni}_{10} \mathrm{Al}_{7} \mathrm{Cu}_{19}$ BMGs as a function of time during annealing at different temperatures

Table 1. Fitting parameters for heat flow during annealing

\begin{tabular}{|c|c|c|}
\hline $\begin{array}{c}\text { Annealing } \\
\text { temperature }\end{array}$ & $W_{0}(\mathrm{~W} / \mathrm{g})$ & $\tau(\mathrm{s})$ \\
\hline $606 \mathrm{~K}$ & 0.031 & 0.943 \\
\hline $616 \mathrm{~K}$ & 0.039 & 0.904 \\
\hline $627 \mathrm{~K}$ & 0.043 & 0.765 \\
\hline $637 \mathrm{~K}$ & 0.044 & 0.635 \\
\hline $648 \mathrm{~K}$ & 0.031 & 0.296 \\
\hline $658 \mathrm{~K}$ & 0.012 & 0.164 \\
\hline
\end{tabular}

The difference in heat flow can also be explained by the energy landscape model. When the annealing temperature is below $\mathrm{Tg}$, the final heat flow, $\delta_{0}$, increases with increasing temperature. This means that additional energy barriers can be surmounted, allowing the system to arrive at the structure with the lowest energy. When the annealing temperature is above $\mathrm{Tg}$, the relaxation mechanism changes. Due to the occurrence of $\alpha$-relaxation, the ergodic configuration on energy landscape is smaller, resulting in a much shorter relaxation time, $\tau$, compared to the relaxation time during the annealing process below $\mathrm{Tg}$, where $\beta$ relaxation is dominant.

In Fig. 2(b), the crystallization peak shifts to lower temperatures after annealing, and at higher temperatures, this is even more apparent. This is due to significant relaxation occurring during the annealing process, especially from the $\alpha$-relaxation at higher temperatures. This relaxation represents a major challenge in glassy physics. Perturbations from some external vital fields can markedly accelerate crystallization. [12] Furthermore, diffusion control, instead of nucleation control, becomes more important in the crystallization process, which leads an increase in the peak width and a reduction in the peak intensity.

Thermal properties of $\mathrm{Zr}_{64-\mathrm{x}} \mathrm{Ni}_{10} \mathrm{Al}_{7} \mathrm{Cu}_{19} \mathrm{Co}_{\mathrm{x}} \mathrm{BMGs}$ have been analyzed by DSC to investigate the effect of composition on the relaxation process. The DSC results show a split peak as a result of the introduction of cobalt. By calculating the enthalpy, one can determine the two peaks correspond to a two-step crystallization process. To determine origin of the two-step crystallization process, we completed electron diffraction and highresolution TEM investigations for two $\mathrm{BMG}$ samples, $\mathrm{Zr}_{64} \mathrm{Ni}_{10} \mathrm{Al}_{7} \mathrm{Cu}_{19}$ and 


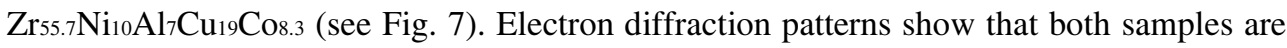
amorphous. However, HRTEM images revealed a nanometer-scaled inhomogeneous structure on the $\mathrm{Zr}_{55.7} \mathrm{Ni}_{10} \mathrm{Al}_{7} \mathrm{Cu}_{19} \mathrm{Co}_{8.3}$ BMG sample that was not present in the $\mathrm{Zr}_{64} \mathrm{Ni}_{10} \mathrm{Al}_{7} \mathrm{Cu}_{19} \mathrm{BMG}$ sample. Phase separation on the scale of $5-10 \mathrm{~nm}$ occurs in the

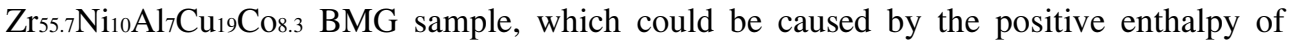
mixing between copper and cobalt. Thus, the normal one step crystallization process splits into two because of the introduction of Co. With increasing of cobalt content, the second crystallization peak is stabilized, whereas the first crystallization peak shifts to lower temperatures. Thus, the relaxation processes occurs over a smaller temperature range as shown in Fig. 3(c).

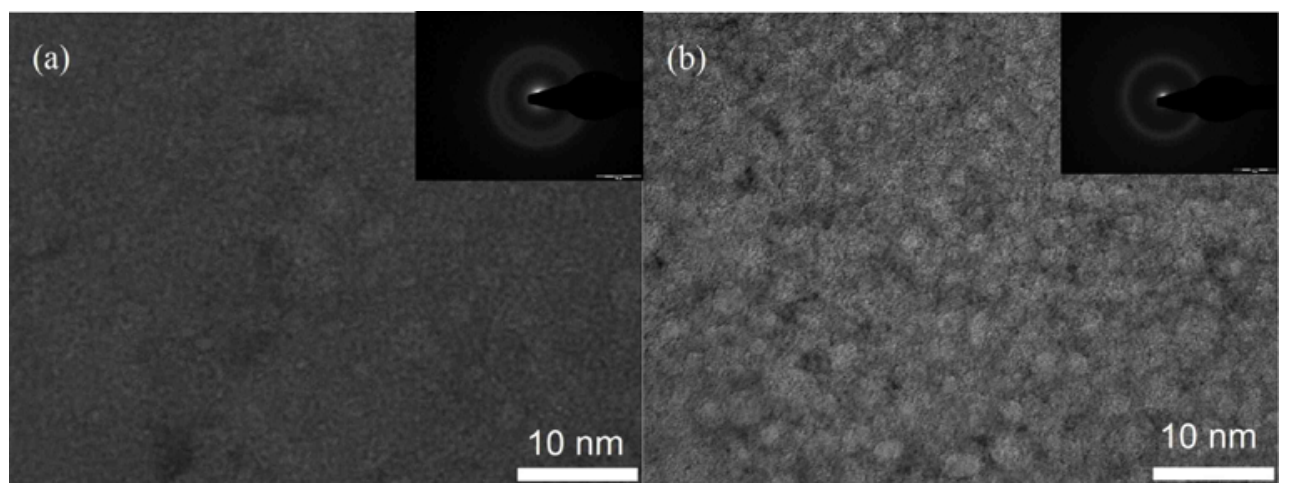

Fig. 7. TEM images of (a) $\mathrm{Zr}_{64} \mathrm{Ni}_{10} \mathrm{Al}_{7} \mathrm{Cu}_{19}$ and (b) $\mathrm{Zr}_{55.7} \mathrm{Ni}_{10} \mathrm{Al}_{7} \mathrm{Cu}_{19} \mathrm{Co} 8.3 \mathrm{BMG}$ samples.

As shown in Fig. 4, annealed BMGs with Co show an obvious difference in relaxation behavior. After low temperature annealing $(563 \mathrm{~K})$, the DSC curve for the BMG with Co shows an endotherm reducing event prior to relaxation that is not present for the annealed BMGs without Co (Fig. 4(d)). This change in $\Delta \mathrm{H}$ can be used to characterize the effects of thermal and mechanical treatments on metallic glasses. For example, heavy plastic deformation or room temperature-liquid nitrogen temperature cycling which introduce nonaffine strains in amorphous structure can lead to a exothermic phenomenon before relaxation, that is termed rejuvenation [13]. In this case, it can be deduced that due to the difference in thermal expansion, non-affine thermal strains arise from the pre-existing heterogeneity in BMGs with Co (see Fig. 7(b)), which leads to the change in $\Delta \mathrm{H}$ visible on DSC curves. It is worth noting that this kind of "rejuvenation" is only in reference to the annealed homogeneous BMGs without Co (Fig. 7(b)), not the as-cast state of the BMGs. This phenomenon disappears when the annealing temperature reaches $583 \mathrm{~K}$ due to the easier diffusion of atoms at higher temperatures. Stress relief annealing occurs. It can also be confirmed by the nanoindentation hardness measurement. It has been reported that hardness of BMGs will increase after annealing due to free volume annihilation [14]. However, Fig. 8 shows that the hardness is instead reduced by the annealing process when the cobalt content reaches $8.3 \%$. The BMGs without cobalt or with low cobalt content have a relatively homogeneous structure and thus show a decrease in free volume during annealing that increases their hardness, as previously reported. The inhomogeneous amorphous structure forms when the cobalt content is at least $8.3 \%$ (see Fig. 7(b)). As discussed earlier, non-affine thermal strains arise during annealing of this structure which is beneficial for the formation free volume, and the hardness decreases. 


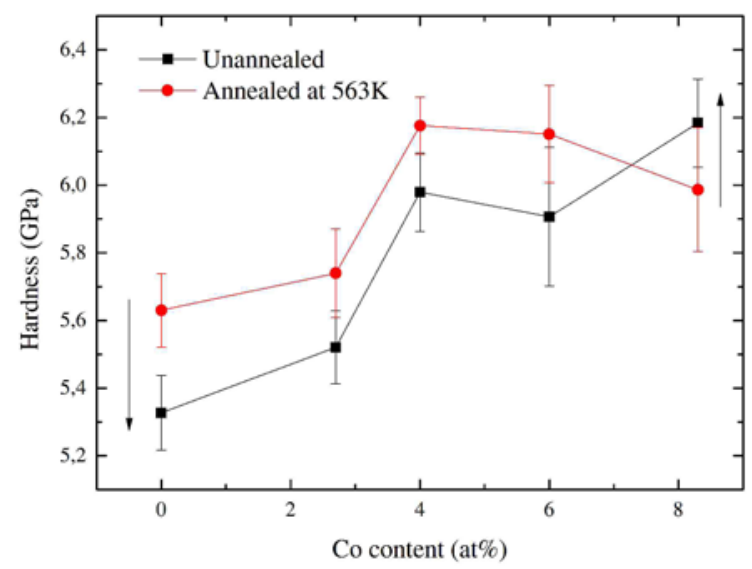

Fig. 8. Hardness of as-cast samples and samples annealed at $563 \mathrm{~K}$

\section{Conclusion}

The effects of annealing and composition on the relaxation and crystallization processes of $\mathrm{Zr}_{64-\mathrm{xNi}} \mathrm{Ni}_{10} \mathrm{Al}_{7} \mathrm{Cu}_{19} \mathrm{Cox}_{\mathrm{x}}$ bulk metallic glasses were studied. The peak at the beginning of relaxation in the DSC curve demonstrates the occurrence of $\beta$-relaxation during annealing; the $\alpha$-relaxation then affects the following crystallization. The introduction of cobalt reduces the temperature range of relaxation and results in a splitting of the crystallization peak into two peaks due to its inhomogeneous amorphous structure. Non-affine thermal strains arise from this structure during low temperature annealing, which leads to an endotherm reducing event before relaxation and a reduction in hardness.

\section{References}

1. A.L. Greer, Y.Q. Cheng, E. Ma, Materials Science and Engineering R 74, 71-132 (2013)

2. L.C. Chen, F. Spaepen, Nature 336, 366-368 (1988)

3. K. Biswas, S. Ram, L. Schultz, J. Eckert, Journal of Alloys and Compounds 397, 104109 (2005)

4. K. Biswas, S. Venkataraman, W.Y. Zhang, S. Ram, J. Eckert, Applied Physics Letter 100, 023501 (2006)

5. S. Ram, G.P. Johari, Philosophical Magazine B 61, 299-310 (1990)

6. T. Matsuyama, N.Terada, T. Baba, T. Sawada, S. Tsuge, K. Wakisaka, S. Tsuda, Journal of Non-Crystalline Solids 198-200, 940-944 (1996)

7. P.S. Frankwicz, S. Ram, H.-J. Fecht, Materials Letters 28, 77-82 (1996)

8. U. Koester, J. Meinhardt, S. Roos, H. Liebertz, Applied Physics Letter 69, 179-181 (1996)

9. YH Liu, G Wang, RJ Wang, DQ Zhao, MX Pan, WH Wang, Science 315, 3185-3188 (2007)

10. G.J. Fan, J.F. Loeffler, R.K. Wunderlich, H.-J. Fecht, Acta Materialia 52, 667-674 (2004)

11. PG Debenedetti, FH Stillinger, Nature 410, 259-267 (2001)

12. HB Yu, WH Wang, K Samwer, Materials Today 16, 183-191 (2013) 
13. S.V. Ketov, Y.H. Sun, S. Nachum, Z. Lu, A. Checchi, A.R. Beraldin, H.Y. Wang, D.V. Louzguine-Luzgin, M.A. Carpenter, A.L. Greer, Nature 524, 200(2015)

14. J Gu, M Song, S Ni, S Guo, Y He, Materials and Design 47, 706-701 (2013) 